\title{
Hypocholesterolemic and Growth Promoting Effects of Lactobacillus plantarum AK Isolated from a Nigerian Fermented Cereal Product on Rats Fed High Fat Diet
}

\author{
B. O. Akanbi ${ }^{*}$, O. O. Agarry \\ Department of Biological Sciences, Faculty of Science, University of Abuja, Abuja, Nigeria

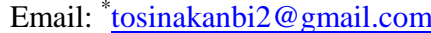

Received December 6, 2013; revised January 6, 2014; accepted January 13, 2014

Copyright (C) 2014 B. O. Akanbi, O. O. Agarry. This is an open access article distributed under the Creative Commons Attribution License, which permits unrestricted use, distribution, and reproduction in any medium, provided the original work is properly cited. In accordance of the Creative Commons Attribution License all Copyrights (C) 2014 are reserved for SCIRP and the owner of the intellectual property B. O. Akanbi, O. O. Agarry. All Copyright (C) 2014 are guarded by law and by SCIRP as a guardian.

\begin{abstract}
The hypocholesterolemic effect of Lactobacillus plantarum AK strain was tested in rats fed high cholesterol diet. The lipid profile, growth rate and microflora dynamics of rats that received $L$. plantarum AK strain were significantly different from the control group $(p<0.5)$. In particular low density lipoprotein was lower in serum of rats that received $L$. plantarum than those of the control group. The test group had a higher growth rate, higher lactobacilli counts but lower coliform and Escherichia coli counts over a period of 40 days and these observed differences were significant $(p<0.05)$. These results are indicative of the abilities of this strain to lower serum cholesterol levels as well as to improve intestinal microbial balance as observed in the microflora dynamics over a period of 40 days.
\end{abstract}

\section{KEYWORDS}

Lactobacillus plantarum; Cholesterol; Growth Promotion; Ogi; Nigeria; Probiotic

\section{Introduction}

Maintenance of cholesterol homeostasis is vital for a healthy status and is achieved through a regulatory network consisting of genes involved in cholesterol synthesis, absorption, metabolism and elimination [1].

Hypercholesterolemia in humans has been shown to play a major role in atherosclerosis, cardiovascular disease, including coronary heart disease and stroke as well as cerebrovascular diseases [2-4]. High cholesterol has also been associated with an increased risk of metabolic syndrome symptoms, including abdominal obesity (large waist circumference), hyperglycemia, hypertriacylglycerolemia and hypertension by as much as 3-fold [5]. Specifically total cholesterol (TC) and low-density lipoprotein cholesterol (LDLC) levels are positively related to coronary heart disease (CHD), and high-density lipoprotein cholesterol (HDLC) levels are negatively related to CHD [6,7], although high TC to HDLC ratio is consi-

*Corresponding author. dered a risk factor [8].

Shaper et al. [9] and Mann and Spoerry [10] are credited with the discovery of hypocholesterolemic effects of diets containing Lactobacillus strains. Recently, there has been renewed interest in lactic acid bacteria (LAB) particularly Lactobacillus spp which are generally recognized as safe (GRAS) and have been linked to their contribution to health such as immunomodulation, anticarcinogenic properties and hypocholesterolemic properties [11-14]. There has been accumulating evidence that the presence Lactobacillus in sufficient amounts is associated with the lowering of serum cholesterol levels in experimental animals $[13,15]$ though this is not always proven in humans [16].

The cholesterol lowering abilities of LAB include assimilation of cholesterol by bacterial cells, deconjugation of bile acids by bacterial acid hydrolases, cholesterol binding to bacterial cell walls, and inhibition of hepatic cholesterol synthesis and/or redistribution of cholesterol from plasma to the liver $[17,18]$. 
LAB in the human gut ferment food-derived indigestible carbohydrates that results in an increased production of short-chain fatty acids (SCFAs) [19]. This may result in decreases in the systemic levels of blood lipids either by inhibiting hepatic cholesterol synthesis or by redistributing cholesterol from plasma to the liver [19]. SCFAs are produced in the large intestine together with smaller amounts of acetate, propionate, and butyrate depending on the substrate [20]. Wolever et al. [21] showed that serum acetate and propionate concentrations were related to serum lipid concentrations in human serum propionate being strongly negatively related to both total and low density (LDL) cholesterol. Removal of cholesterol occurs because deconjugated bile acids are not well absorbed by the gut mucosa and are excreted through the feces and urine. The excretion of bile acids results in decreased enterohepatic recirculation and therefore more cholesterol, which is the precursor of bile acids is utilized for de novo bile acid synthesis.

In Nigeria, fermented foods of plant origin are popular but the hypocholesterolemic effect of Lactobacillus spp isolates of these foods has not been adequately studied. The present study was conducted to study the hypocholesterolemic effect of one strain from a common fermented food in vivo.

\section{Materials and Methods}

Lactobacillus plantarum AK was isolated from Ogi one of the fermented cereals produced from maize, sorghum or millet. It is usually consumed as a porridge in all regions of Nigeria. This isolate was obtained from ogi produced from millet (Pennisetumglaucum). The isolate was identified based on the pattern of carbohydrate fermentation (Biomerieux) and had also shown tolerance to acidic $\mathrm{pH}$ values as well as bile tolerance (unpublished data).

L. plantarum AK was grown in Mann Rogosa and Sharpe (MRS) broth (Oxoid, Basingstoke) at $37^{\circ} \mathrm{C}$ for 18 h. Broth culture samples were withdrawn periodically and the optical density (OD) measured in spectrophotometer $(600 \mathrm{~nm})$. Serial dilutions were pour plated on MRS agar in triplicates and incubated for $48 \mathrm{~h}$ at $37^{\circ} \mathrm{C}$. The values were used to produce a standard curve by plotting OD versus $\mathrm{cfu} / \mathrm{ml}$. From the calibration curve, the OD equivalent to $2.0 \times 10^{9} \mathrm{cfu} / \mathrm{ml}$ was determined.

\subsection{Animal Feeding and Grouping}

Forty, two weeks old pathogen free male Wister albino rats with similar weights were obtained from the National Institute for Trypanosomal Research Kaduna, Nigeria. Relatively young animals were used in order to clearly assess the impact on growth and microfloral changes. The diet consisted of $75 \%$ basic diet (protein $20 \%$, fat
$10 \%$, fibre 10\%, $2800 \mathrm{Kcal} . / \mathrm{kg}$, vitamins and minerals), $10 \%$ lard, $10 \%$ soybean meal and 5\% egg yolk to create a hyperlipidemic model as described by Wang et al. [22].

Environmental conditions at the animal facility unit of the department of biological sciences where the rats were housed were well controlled. Temperature was maintained at $25^{\circ} \mathrm{C} \pm 1{ }^{\circ} \mathrm{C}$ and humidity at $60 \%$ and in a cycle of $12 \mathrm{~h}$ of light and $12 \mathrm{~h}$ of darkness. The rats were divided into two groups of twenty each and housed individually: test group A, fed with high-cholesterol diet and $L$. plantarum and control, group B fed with high-cholesterol diet only. Each rat in group A was administered by gavage a dose of $2.0 \times 10^{9} \mathrm{cfu} / \mathrm{ml}$ of the organism in sterile physiological saline per day. The care and use of animals were in accordance with institutional and national standards.

Food and water were accessible at all times and were replenished every day. The animals were fed for 40 days, during which time body weight and food intake were recorded. During this period, blood was obtained at 10 day intervals for serum cholesterol and triglycerides analysis. Every ten days, two animals were randomly chosen from each of the two groups fasted for $12 \mathrm{hr}$ and euthanized using diethyl ether. Freshly voided rat faecal material was also collected every ten days for bacterial counts. After the feeding period, the remaining rats, fourteen in number were fasted for 12-hr and euthanized and were presented for tests as above.

\subsection{Assay for Fecal Microflora}

The enumeration of bacteria was done by aseptically collecting freshly voided feces from all treatment groups in sterile tubes on the first day, $10^{\text {th }}$ day and subsequently every ten days thereafter. Samples from all animals in a group were collected. Ten grams of each sample was homogenized using $90 \mathrm{ml}$ sterile physiological saline as diluent and subsequent tenfold serial dilutions of each sample were plated in triplicate. For coliform counts, eosine methylene blue agar (EMB) (Oxoid Basingstoke), EMB was used for enumeration whereas Man rogosa and sharpe agar (Oxoid Basingstoke) MRS was used to enumerate lactobacilli using anaerobic jar (Oxoid Basingstoke). These were incubated at $37^{\circ} \mathrm{C}$ for 24 -h for coliforms and 48-h for lactobacilli.

\subsection{Determination of Serum Lipid Profile}

Four milliliters of the blood samples were transferred to non-heparinized vacuum collection tubes. The tubes were allowed to stand in a test tube rack at $0^{\circ} \mathrm{C}$ for 30 minutes, and then centrifuged at $2000 \times \mathrm{g}$ for 15 minutes at $4^{\circ} \mathrm{C}$. Lipid profile analyses were performed spectrophotometrically using spectrumlab (Easy Way Medical, England). 


\subsection{Statistical Analysis}

T-Test was used to compare the means of the initial and final weights of test and control groups as well as the lipid profile of the serum from both groups using Microsoft Excel (version 2010). Fecal microbial counts at different sampling times were compared using ANOVA (SPSS 16.0 (SPSS Inc., Chicago, IL, USA) and means were separated using Duncan's Post Hoc Test. Differences in means were considered significant if $p \leq 0.05$.

\section{Results}

Table 1 provides a summary of growth performance and the statistical significance. The average initial body weights of the two groups of 20 each were only slightly different and were not statistically different $(\mathrm{p}>0.05)$. The final average body weight of the test group (282.86 g) which received Lactobacillus plantarum was higher than the control group which did not receive the organism (258.14 g). The observed differences in final average body weight, body weight gain and food intake were all significantly different $(\mathrm{p}<0.05)$.

Table 2 shows the lipid profile of test and control rats as well as the statistical inference of these results. In all the periods i.e. day 10, 20, 30 and 40, the values of low-density lipoprotein cholesterol were generally higher in the control group and the observed differences were statistically significant $(\mathrm{p}<0.05)$. The values in highdensity lipoprotein cholesterol were generally higher in control group and the observed difference were also significant $(p<0.05)$. Total cholesterol values for the two groups were not significantly different in the first 20 days ( $p>0.05)$. However the values for the last 20 days were significantly different $(\mathrm{p}<0.05)$.

Figure 1 shows the log count of fecal coliforms in the test (A) and control (B) groups. Error bars and letters indicate standard deviations and significant differences or otherwise respectively. Bars with similar letters are not significantly different whereas different letters indicate statistically significant differences. The administration of L. plantarum showed an effect on the total coliform counts when the counts of the two groups were compared. The test group initially had a significantly higher count on the first day and also day 10 than the control group at $5.85 \log _{10} \mathrm{cfu} / \mathrm{g}$ and $5.89 \log _{10} \mathrm{cfu}$ respectively compared to $5.26 \log _{10} \mathrm{cfu} / \mathrm{g}$ and $5.10 \log _{10} \mathrm{cfu} / \mathrm{g}$ respectively. These values dropped during subsequent

Table 1. Body weight gain, total food intake, and food efficiency of rats fed on high-cholesterol diet.

\begin{tabular}{ccccc}
\hline Parameter & Group A (Test) & Group B (Control) & p value & $\begin{array}{c}\text { Statistical significance } \\
\text { at 95\% probability level }\end{array}$ \\
\hline Initial body weight (g) & $44.48 \pm 0.25$ & $44.60 \pm 0.18$ & 0.1 & P $>0.05$, initial body weights not significantly different \\
Final body weight (g) & $282.86 \pm 7.54$ & $258.14 \pm 4.22$ & 0.000 & P $<0.05$, final body weights significantly different \\
Body weight gain (g/40 days) & $235.79 \pm 4.61$ & $213 \pm 5.42$ & 0.000 & P < 0.05, body weight gain significantly different \\
Food intake (g/40 days) & $1585.57 \pm 7.6$ & $1561.57 \pm 11.91$ & 0.000 & P $<0.05$, food intake significantly different \\
Average Food efficiency (\%) & 14.83 & 13.70 & & \\
\hline
\end{tabular}

Values are average \pm standard deviation.

Table 2. Serum lipids contents of rats fed on high-cholesterol diet.

\begin{tabular}{cccccc}
\hline Period & Type of lipid & Day 10 & Day 20 & Day 30 & Day 40 \\
\hline Group A & LDLPC & $1.72 \pm 0.014$ & $1.43 \pm 0.04$ & $1.22 \pm 0.02$ & $0.85 \pm 0.01$ \\
Group B & LDLPC & $1.83 \pm 0.01$ & $1.96 \pm 0.014$ & $2.46 \pm 0.01$ & $2.88 \pm 0.01$ \\
p value & & $0.009^{*}$ & $0.003^{*}$ & $0.0002^{*}$ & $0.0000^{*}$ \\
Group A & HDLPC & $0.84 \pm 0.01$ & $1.13 \pm 0.03$ & $1.05 \pm 0.03$ & $1.23 \pm 0.02$ \\
Group B & HDLPC & $1.47 \pm 0.02$ & $1.64 \pm 0.03$ & $2.36 \pm 0.03$ & $2.91 \pm 0.01$ \\
p value & & $0.0008^{*}$ & $0.003^{*}$ & $0.0005^{*}$ & $0.0001^{*}$ \\
Group A & TC & $2.05 \pm 0.08$ & $3.50 \pm 0.00$ & $3.70 \pm 0.08$ & $3.22 \pm 0.01$ \\
Group B & TC & $2.12 \pm 0.04$ & $3.78 \pm 0.120$ & $4.12 \pm 0.070$ & $4.65 \pm 0.07$ \\
p value & & 0.21 & 0.3 & $0.03^{*}$ & $0.0000^{*}$ \\
Group A & TTG & $1.56 \pm 0.02$ & $2.18 \pm 0.04$ & $3.17 \pm 0.01$ & $2.54 \pm 0.06$ \\
Group B & TTG & $1.75 \pm 0.021$ & $2.83 \pm 0.08$ & $3.34 \pm 0.00$ & $3.41 \pm 0.11$ \\
p value & & $0.01^{*}$ & $0.01^{*}$ & $0.003^{*}$ & $0.008^{*}$ \\
\hline
\end{tabular}

Values are in Millimole per liter; Key: LDLPC; Low-density lipoprotein cholesterol, HDLPC; High-density lipoprotein cholesterol, TC; Total Cholesterol, TTG; Total triglycerides; ${ }^{*}$ indicate means are significantly different between groups at $\mathrm{p}<0.05$. 


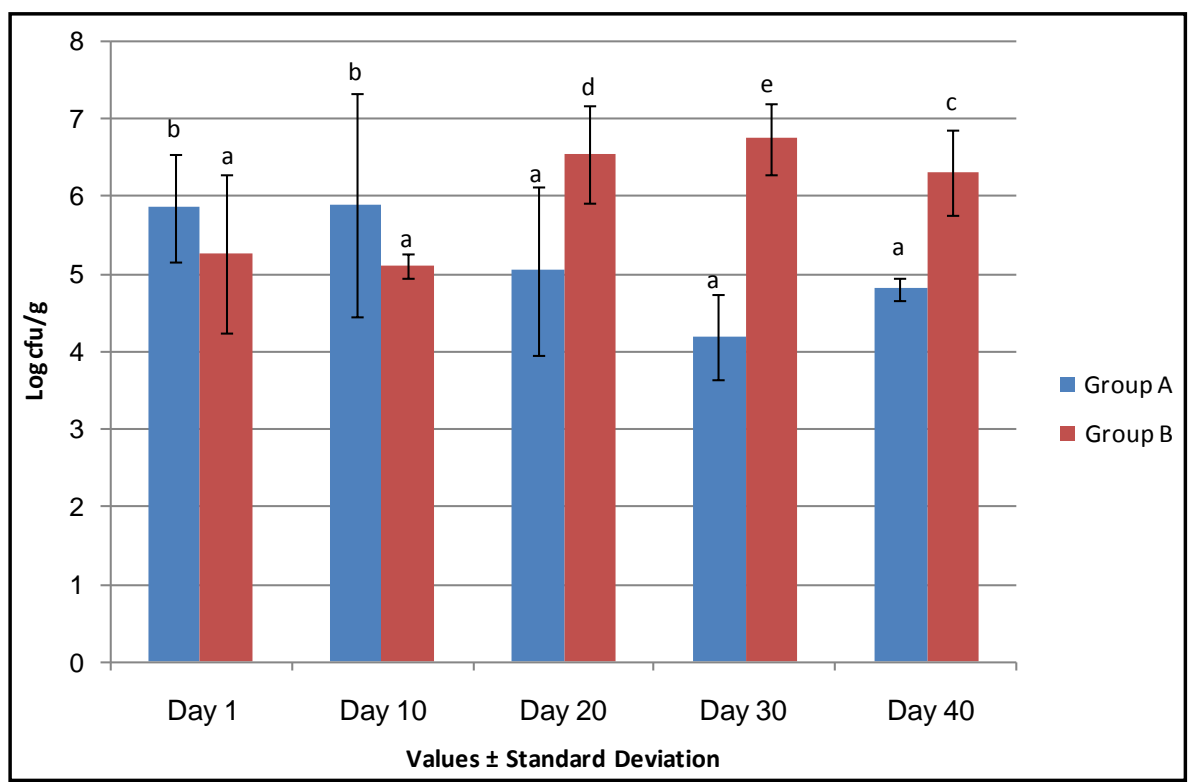

Figure 1. Rat fecal coliform count on EMB agar. Values are means \pm standard deviation, means with different letters significantly different $(\mathrm{p}<\mathbf{0 . 0 5})$.

sampling periods in the test group. Statistical analysis indicated that the coliform numbers obtained for group A(test group) for days 20, 30 and 40 were similar and were also significantly different from those of group B i.e. control group ( $\mathrm{p}<0.05)$. For the control group, coliform numbers were statistically similar in the first 20 days and different subsequently.

Figure 2 shows the fecal lactobacilli count of the two groups of rats as explained previously. Error bars and letters are also as earlier explained in Figure 1. Values of bacterial counts are means \pm standard deviation. Means with different letters indicate significant difference ( $\mathrm{p}<$ 0.05). The counts of group A, administered L. plantarum and group $\mathrm{B}$ were similar in the first day $(\mathrm{P}<0.05)$. On the tenth day the counts of group $\mathrm{A}$ was higher than group B at $6.7 \mathrm{log} \mathrm{cfu} / \mathrm{g}$ and $5.43 \mathrm{log} \mathrm{cfu} / \mathrm{g}$ respectively. The observed differences were however not significantly different ( $>0.05$ ). The counts for group A at 20 days, 30 days and 40 days were $8.6 \mathrm{cfu} / \mathrm{g}, 8.78 \mathrm{cfu} / \mathrm{g}$ and 8.56 $\mathrm{cfu} / \mathrm{g}$ respectively and these values were significantly higher than the corresponding values of group B which were $6.57 \mathrm{cfu} / \mathrm{g}, 6.45 \mathrm{cfu} / \mathrm{g}$ and $6.42 \mathrm{cfu} / \mathrm{g}$ respectively during the same period. Overall, the lactobacilli counts of group B were not significantly different throughout the duration of experiment.

\section{Discussion}

The administration of $L$. plantarum had a significant effect on weight of the rats as the rats which received the organism had significantly higher weights compared to the control group. The feed consumption as well as feed efficiency was also significantly higher in the test group.
These could be due to effects on general health, alteration of microbial balance or other unknown factors. These results appear not to be supported by some other studies such as Chiu et al. [15] where all groups of hamsters fed high cholesterol diet showed no significant differences in body weight gain, total food intake and food efficiency irrespective of administration of lactobacilli or not. Similarly Wang et al. [13] found no significant differences in body weight gain; food intake and food efficiency in animals supplemented with a strain of $L$. plantarum and the control group fed high lipid diets. In support of this study, Xie et al. [23] found that the administration of lactobacilli significantly improved weight again and food efficiency compared to the control group in a similar study. However, food intake was unaffected in their study unlike what obtained in the present study.

The consumption of $L$. plantarum had a significant effect on the lipid profile of the rats. Low-density lipoprotein cholesterol was generally higher in the control group and the observed differences were statistically significant $(\mathrm{P}<0.05)$. This is in agreement with other studies such as Wang et al. [22] that studied the effects of two L. plantarum strains in hyperlipidemic mice. In their study, the total cholesterol, triglycerides and low-density lipoprotein cholesterol levels of mice in the control group were found to be significantly higher. Similar results were reported by Huang et al. [24] and Wang et al. [13] from studies on cholesterol-lowering effects of $L$. plantarum strains isolated from Tibetan kefir grains. Huang et al. [24] found that their isolate significantly lowered serum total cholesterol, low-density lipoprotein cholesterol, and triglycerides concentrations but not serum high-density lipoprotein cholesterol concentrations and furthermore 


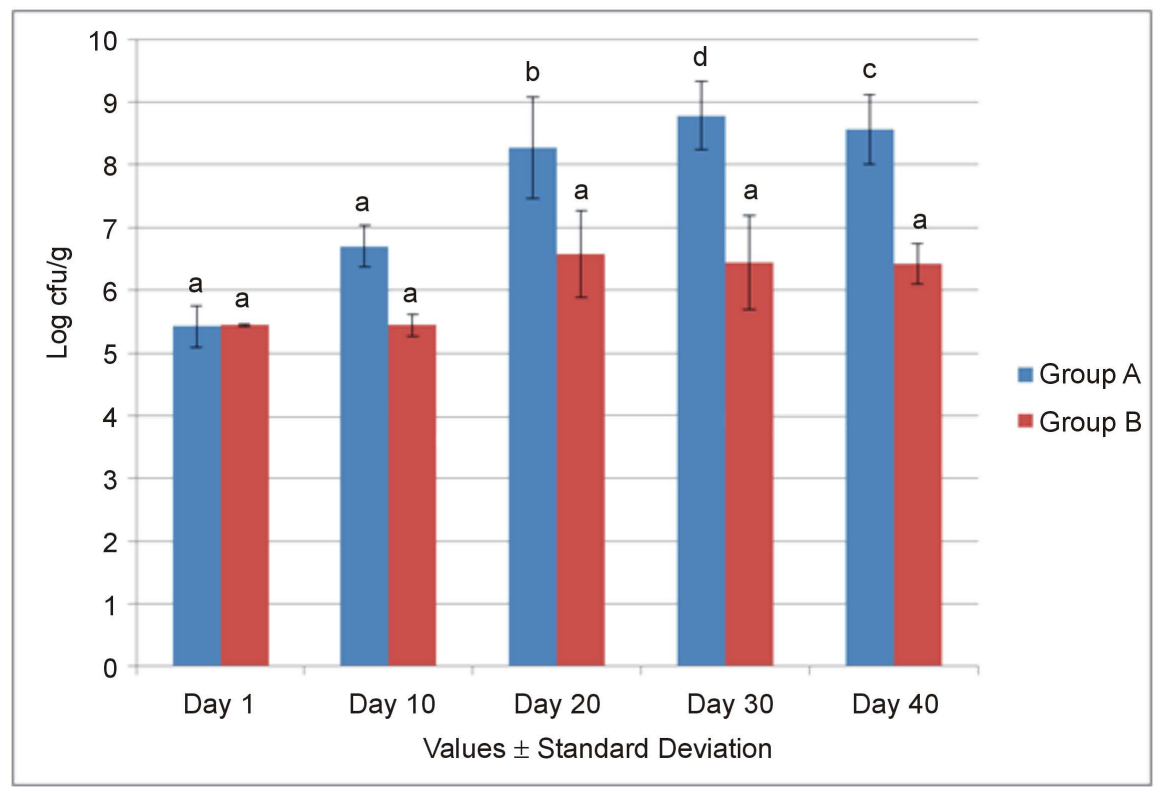

Figure 2. Rat fecal lactobacilli count on MRS agar.

elucidated the mechanism by noting inhibition of cholesterol absorption through down regulation of NiemannPick C1-like 1 (NPC1L1) expression in Caco-2 cells after a period of four weeks. Studies of other Lactobacillus spp have also demonstrated hypocholesterolemic effects similar to these, such as Chiu et al. [15], Xie et al. [23] indicating that these effects are not limited to a few species.

The cholesterol lowering abilities of LAB generally has been observed to include assimilation of cholesterol by bacterial cells, deconjugation of bile acids by bacterial acid hydrolases cholesterol binding to bacterial cell walls, and inhibition of hepatic cholesterol synthesis and/or redistribution of cholesterol from plasma to the liver $[17,18]$.

For L. plantarum, recent studies have provided more insights such as the mechanism previously elucidated by Huang et al. [24] where the expression of NPC1L1 in the duodenum and jejunum was significantly decreased following $L$. plantarum feeding. Additionally Wang et al. [23] have hypothesized that $L$. plantarum significantly increased liver and kidney Nuclear factor-erythroid 2-related factor 2 (Nrf2) expression, which is a transcription factor that binds to antioxidant response elements (ARE) in the promoter regions of many anti-oxidative enzymes and phase II detoxifying enzymes [25]. According to this hypothesis, accumulation of Nrf2 in the liver may initiate anti-oxidative stress and result in inhibition of $\mathrm{Ox}$ Low-density lipoprotein cholesterol production with the remarkable modifications in antioxidant defense mechanisms seen in their in vivo study. It is likely that any or a combination of these models is also operational in this study.
The administration of $L$. plantarum significantly reduced the total coliform counts of the test groups. Other studies have shown variable results ranging from no significant effects such as the study by Wang et al. [13] to significant increases in E. coli population which is a component of the coliform group in groups administered lactobacilli as observed by Xie et al. [23]. A generally accepted explanation of probiotic action of lactic acid bacteria include improved intestinal microflora balance as seen in the present study, survival in stomach, bile salts and colonization of the large bowel. The strain used in this study had previously shown good survival in conditions simulating normal exposure to stomach acidity as well as high bile salt concentrations and additionally large bowel colonization can be inferred from the high counts of lactobacilli in the test group compared to the control group. The lactobacilli count increased steadily with time in the course of the study in agreement with several studies where administration of lactobacilli is positively correlated with significantly higher fecal lactobacilli counts $[13,15,23]$. In these studies the control is usually stable as in the present study. The difference at the final stage was about $2 \mathrm{log} \mathrm{cfu} / \mathrm{g}$ in this study which is agreement with other studies that reported figures between 1 - $3 \log \mathrm{cfu} / \mathrm{g}[13,15,23]$.

\section{Conclusion}

Most previous studies have focused on lactobacilli isolated from either dairy products or healthy human feces but this study is significant in the sense that the source of the organism is plant based. The results of this study have indicated that the L. plantarum strain used can low- 
er low-density lipoprotein cholesterol, total Cholesterol as well as total triglycerides. In addition, it may modify microbial balance in the gut since it appeared to have significantly lowered coliform populations. These are positive attributes that could be potentially explored for use in human health. The age of the rats also indicates that it is a suitable model to use to study microflora dynamics as well as impact of growth presumably because the microflora is still developing at this stage. These observations and hypothesis need to be validated through more in-depth studies and there is also the imperative to study the mechanism(s) of lipid metabolism in this isolate.

\section{REFERENCES}

[1] R. Deng, "Food and Food Supplements with Hypocholesterolemic Effects," Recent Patents on Food, Nutrition \& Agriculture, Vol. 1, No. 1, 2009, pp. 15-24. http://dx.doi.org/10.2174/2212798410901010015

[2] D. Steinberg, "Thematic Review Series: The Pathogenesis of Atherosclerosis. An Interpretive History of the Cholesterol Controversy: Part II: The Early Evidence Linking Hypercholesterolemia to Coronary Disease in Humans,” Journal of Lipid Research, Vol. 46, No. 2, 2005, pp. 179190. http://dx.doi.org/10.1194/jlr.R400012-JLR200

[3] C. Ballantyne, B. Arroll and J. Shepherd, "CVD Management: Towards a Global Consensus,” European Heart Journal, Vol. 22, No. 21, 2005, pp. 2224-2231.

[4] J. Lee, Y. Kim, H. S. Yun, J. G. Kim, S. Oh and S. H. Kim, "Genetic and Proteomic Analysis of Factors Affecting Serum Cholesterol Reduction by Lactobacillus acidophilus A4,” Applied and Environmental Microbiology, Vol. 76, No. 14, 2010, pp. 4829-4835. http://dx.doi.org/10.1128/AEM.02892-09

[5] B. Isomaa, P. Almgren, T. Tuomi, G. Forsen, K. Lahti, M. Nissen, M. Taskinen and L. Groop, "Cardiovascular Morbidity and Mortality Associated with the Metabolic Syndrome,” Diabetes Care, Vol. 24, No. 4, 2001, pp. 683689. http://dx.doi.org/10.2337/diacare.24.4.683

[6] L. Kritharides, "Reducing Low-Density Lipoprotein Cholesterol-Treating to Target and Meeting New European Goals,” European Heart Journal, Vol. 6, 2004, pp. A12A18.

[7] V. Sblendorio and B. Palmieri, “Accuracy of Analyses for Lipid Profile Parameters as Measured with the CR3000 System," European Review for Medical and Pharmacological Sciences, Vol. 12, No. 3, 2008, pp. 191-196.

[8] B. J. Arsenault, J. S. Rana, E. S. G. Stroes, J. Després, P. K. Shah, J. J. P. Kastelein, N. J. Wareham, S. M. Boekholdt and K. Khaw, "Beyond Low-Density Lipoprotein Cholesterol: Respective Contributions of Non-High-Density Lipoprotein Cholesterol Levels, Triglycerides, and the Total Cholesterol/High-Density Lipoprotein Cholesterol Ratio to Coronary Heart Disease Risk in Apparently Healthy Men and Women,” Journal of the American College of Cardiology, Vol. 55, No. 1, 2010, pp. 35-41.

[9] A. G. Shaper, K. W. Jones and J. Kyobe, "Serum Lipids in the Three Nomadic Tribes of Northern Kenya," The
American Journal of Clinical Nutrition, Vol. 13, 1963, pp. 135-146.

[10] G. V. Mann and A. Spoerry, "Studies of a Surfactant and Cholesteremia in the Maasai," The American Journal of Clinical Nutrition, Vol. 27, No. 5, 1974, pp. 464-469.

[11] M. Saarela, G. Mogensen, R. Fonden, J. Matto and T. Mattila-Sandholm, "Probiotic Bacteria: Safety, Functional and Technological Properties,” Journal of Biotechnology, Vol. 84, No. 3, 2000, pp. 197-215. http://dx.doi.org/10.1016/S0168-1656(00)00375-8

[12] G. Zoumpopoulou, B. Foligne, K. Christodoulou, C. Grangette, B. Pot and E. Tsakalidou, "Lactobacillus fermentum ACA-DC 179 Displays Probiotic Potential in Vitro and Protects against Trinitrobenzene Sulfonic Acid (TNBS)Induced Colitis and Salmonella Infection in Murine Models,” International Journal of Food Microbiology, Vol. 121, No. 1, 2008, pp. 18-26.

[13] Y. Wang, N. Xu, A. Xi, Z. Ahmed, B. Zhang and X. Bai, "Effects of Lactobacillus plantarum MA2 Isolated from Tibet Kefir on Lipid Metabolism and Intestinal Microflora of Rats Fed on High-Cholesterol Diet," Applied Microbiology and Biotechnology, Vol. 84, No. 2, 2009, pp. 341-347. http://dx.doi.org/10.1007/s00253-009-2012-x

[14] T. Asahara, K. Shimizu, T. Takada, S. Kado, N. Yuki, M. Morotomi, R. Tanaka and K. Nomoto, "Protective Effect of Lactobacillus caseistrain Shirota against Lethal Infection with Multi-Drug Resistant Salmonella entericaserovar Typhimurium DT104 in Mice,” Journal of Applied Microbiology, Vol. 110, No. 1, 2010, pp. 163-173. http://dx.doi.org/10.1111/j.1365-2672.2010.04884.x

[15] C. Chiu, T. Lu, Y. Tseng and T. Pan, "The Effects of Lactobacillus-Fermented Milk on Lipid Metabolism in Hamsters Fed on High-Cholesterol Diet," Applied Microbiology and Biotechnology, Vol. 71, No. 2, 2006, pp. 238-245. http://dx.doi.org/10.1007/s00253-005-0145-0

[16] G. Jahreisa, H. Vogelsangb, G. Kiesslinga, R. Schuberta, C. Buntec, W. P. Hammes, "Influence of Probiotic Sausage (Lactobacillus paracasei) on Blood Lipids and Immunological Parameters of Healthy Volunteers,” Food Research International, Vol. 35, No. 2-3, 2002, pp. 133138.

[17] I. De Smet, P. De Boever and W. Verstraete, “Cholesterol Lowering in Pigs through Enhanced Bacterial Bile Salt Hydrolase Activity,” British Journal of Nutrition, Vol. 79, No. 2, 1998, pp. 185-194.

[18] J. Lovegrove and K. Jackson, “Coronary Heart Disease,” In: T. Mattila Sandholm and M. Saarela, Eds., Functional Dairy Products, Woodhill Publishing, Cambridge, 2003, pp. 54-87.

[19] D. I. A. Pereira and G. R. Gibson, "Effects of Consumption of Probiotics and Prebiotics on Serum Lipid Levels in Humans," Critical Reviews in Biochemistry and Molecular Biology, Vol. 37, No. 4, 2002, pp. 259-281. http://dx.doi.org/10.1080/10409230290771519

[20] M. P. St-Onge, E. R. Farnworth and P. J. H. Jones, "Consumption of Fermented and Unfermented Dairy Products: Effects on Cholesterol Concentrations and Metabolism," The American Journal of Clinical Nutrition, Vol. 71, No. 3, 2000, pp. 674-681. 
[21] T. M. S. Wolever, J. Fernandes and A. Veketeshwer Rao, "Serum Acetate: Propionate Ratio Is Related to Serum Cholesterol in Men but Not in Women," The Journal of Nutrition, Vol. 126, No. 11, 1996, pp. 2790-2797.

[22] L. Wang, K. Liu, D. Gao and J. Hao, "Protective Effects of Two Lactobacillus plantarum Strains in Hyperlipidemic Mice,” World Journal of Gastroenterology, Vol. 19, No. 20, 2013, pp. 3150-3156. http://dx.doi.org/10.3748/wjg.v19.i20.3150

[23] N. Xie, Y. Cui, Y. Yin, X. Zhao, J. Yang, Z. Wang, N. Fu, Y. Tang, X. Wang, X. Liu, C. Wang and F. Lu, "Effects of Two Lactobacillus Strains on Lipid Metabolism and Intestinal Microflora in Rats Fed a High-Cholesterol Diet,”
BMC Complementary and Alternative Medicine, Vol. 11, No. 53, 2011, pp. 1-11.

[24] Y. Huang, F. Wu, X. Wang, Y. Sui, L. Yang and J. Wang, "Characterization of Lactobacillus plantarum Lp27 Isolated from Tibetan Kefir Grains: A Potential Probiotic bacterium with Cholesterol-Lowering Effects," Journal of Dairy Science, Vol. 96, No. 5, 2013, pp. 2816-2825. http://dx.doi.org/10.3168/jds.2012-6371

[25] K. Itoh, T. Ishii, N. Wakabayashi and M. Yamamoto, "Regulatory Mechanisms of Cellular Response to Oxidative Stress,” Free Radical Research, Vol. 31, No. 4, 1999, pp. 319-324.

http://dx.doi.org/10.1080/10715769900300881 